\title{
User Experience and usability of a new virtual reality set-up to treat eating disorders: a pilot study
}

1 Marta Matamala-Gomez ${ }^{1}$, Eleonora Brivio ${ }^{2}$, Alice Chirico $^{2}$, Clelia Malighetti ${ }^{2}$, Olivia Realdon ${ }^{1}$, 2 Silvia Serino ${ }^{3}$, Antonios Dakanalis ${ }^{4,5}$, Giulia Corno $^{6}$, Nicoletta Polli ${ }^{7,8}$, Chiara Cacciatore, 3 Giuseppe Riva ${ }^{2,9}$, Fabrizia Mantovani ${ }^{1}$

$4{ }^{1}$ University of Milano-Bicocca, "Riccardo Massa" Department of Human Sciences for Education, Milan, 5 Italy.

$6 \quad{ }^{2}$ Università Cattolica del Sacro Cuore, Departament of Psychology, Milan, Italy.

$7 \quad{ }^{3}$ Lausanne University Hospital (CHUV), MySpace Lab, Lausanne, Switzerland.

$8{ }^{4}$ Department of Medicine and Surgery, University of Milano Bicocca, Milan, Italy.

$9 \quad{ }^{5}$ Department of Brain and Behavioral Sciences, University of Pavia, Pavia, Italy.

$10{ }^{6}$ Université du Québec en Outaouais · Département de psychoéducation et de psychologie, Québec, Canada.

117 'Istituto Auxologico Italiano (IRCCS), 'UO di Endocrinologia e Malattie Metaboliche', Milan, Italy.

$12{ }^{8}$ University of Milan, 'Dipartimento di Scienze Cliniche e di Comunità', Milan, Italy.

$13{ }^{9}$ Applied Technology for Neuro-Psychology Laboratory, Istituto Auxologico Italiano, IRCCS, Milan, Italy.

14 * Correspondence:

15 Marta Matamala-Gomez

16 marta.matamala10@gmail.com

17 marta.matamalagomez@unimib.it

18 Keywords: virtual reality, embodiment, usability, user experience, anorexia nervosa. 


\section{Abstract}

25 Virtual Reality (VR) has progressively emerged as an effective tool for wellbeing and health in clinical 26 populations. VR effectiveness has been tested before in Anorexia Nervosa (AN) with full-body 27 illusion. It consists in the embodiment of patients with AN into a different virtual body to modify their 28 long-term memory of the body as a crucial factor for the onset and maintenance of this disorder. We 29 extended this protocol using the autobiographical recall emotion-induction technique, in which patients 30 recall an emotional episode of their life related to their body. In this pilot study, we aimed to test the 31 usability and User Experience (UX) of this VR-based protocol. Five Italian women with AN were 32 embodied in a virtual body resembling their perceived body size from an ego- and an allocentric 33 perspective while remembering episodes of their life related to their body. High levels of embodiment 34 were reported while embodied in a virtual body resembling their real perceived body size for ownership $35(\mathrm{p}<0.0001)$, agency $(\mathrm{p}=0.04)$, and self-location $(\mathrm{p}=0.023)$. Negative affective state increase after 36 session $2(\mathrm{p}=0.012)$, and positive affective state increase after session $4(\mathrm{p}=0.006)$ (PANAS). However, 37 further iteration of the VR system is needed to improve user experience and usability of the system.

38 Keywords: virtual reality, embodiment, usability, user experience, anorexia nervosa. 
Virtual reality (VR) can be defined as a computer-generated reality in which presence is a key factor to perceive the generated virtual environment as real (Riva et al., 2019). The sense of presence is the feeling of being physically immersed in a computer-generated environment and perceiving of being able to interact with the events happening in that virtual environment that can modulate users' affective responses (Riva et al., 2007; Slater, 2009). Due to the lowering cost of VR equipment and the validated effectiveness of VR in modulating users' physical and emotional responses, VR systems are increasingly being used for promoting well-being in different clinical populations (Montana et al., 2020; Parsons, 2015).

Particularly, today there is an increasing interest in the use of VR to modulate the mental body representation of healthy and clinical populations by means of body-illusions, that is the illusion of perceiving a fake body as if this was the real one (Matamala-Gomez et al., 2018, 2020b; Serino et al., 2019). For instance, body illusions have been targeted towards specific body parts, such as in the case of the rubber hand illusion (RHI) (Botvinick and Cohen, 1998). In the RHI study participants experienced ownership illusion over a fake body part -a rubber hand- while the experimenter synchronously stimulated both the real (outside their field of view), and rubber hand (inside their field of view) by using tactile stimulation (visuo-tactile stimulus). Thus, by using synchronous visuo-tactile stimulation it is also possible to induce ownership over a fake limb, and even over an entire full body, creating a full body illusion (FBI) (Maselli and Slater, 2013). Furthermore, in VR, agency (the feel of controlling the virtual body movements) over the virtual body can be also induced by giving a synchronous visuomotor feedback to the users, feeling agency over the actions of the virtual body into the immersed virtual environment (Sanchez-Vives et al., 2010).

In addition to body ownership illusions, the body-swap illusion has been also investigated by using entire fake bodies (a mannequin) through the use of synchronous visuo-tactile stimulations to the real and fake bodies (Petkova and Ehrsson, 2008). In the study by Petkova and Ehrsson, participants had the perceptual illusion of being localized in another persons' body, or an artificial humanoid body that was physically placed in front of them as belonging to themselves inducing a "body-swap" illusion. Thus, in order to induce such body-swap illusion, (meaning the change of the participants center of awareness from an egocentric (own body) to a allocentric (another body)) the authors described the following criteria which must be occurring at the same time: a) the use of a synchronous visual and somatosensory feedback about the state of the body, b) the use of a fake body with enough human 
features, and c) the adoption/use of a first-person perspective of the fake body. In this concern, other investigations have shown that it is possible to modify the body image in patients with AN over an entire virtual body to decrease body-size overestimation (Keizer et al., 2016). Through the of virtual bodies it is possible to measure the conscious internal representation of one's body appearance allowing participants to compare the represented virtual body to their ideal body appearance and to their real body appearance (Neyret et al., 2020). Moreover, Serino and colleagues (2019) used the body-swap illusion in patients with AN, to evaluate possible changes in their distorted body representations. These results are coherent with many studies that demonstrate the power of VR-based body illusions and embodiment in another body to change behavioral and physiological responses (Riva et al., 2019; Slater and Sanchez-Vives, 2016).

However, although the use of body illusions through VR systems is growing in clinical applications, few studies aimed to investigate the usability and user experience while using VR systems for clinical purposes. To this aim, in the present study we investigate the usability and user experience of a VRbased system directed to modify the mental body representation of patients with AN through a full virtual body illusion and body-swapping in VR.

\section{Background. State of art}

\subsection{Virtual reality for positive change}

Today, the use of technology for human-computer interaction to improve users' quality of life has been growing (Riva et al., 2012). One of the most important aims in the use of technology in clinical applications is the increase of happiness and psychological well-being (Gaggioli et al., 2016). In this regard, the recently emerged field of positive psychology that refers to the bio-psycho-social aspects of cognitions, emotions, and positive experiences (Burgess, 2019; Seligman and Csikszentmihalyi,

103 2000), has been paving the way to the development of new technology systems directed to improve the quality of personal experience, leading to the enhancement of well-being of the users (Botella et al., 2012). Particularly, the positive psychology approach aims to develop new interactive systems that 1) enhance positive emotional states through "hedonic technologies", 2) facilitate engagement and sense of flow through the use of "eudaimonic technologies", and 3) promote social interaction and connectedness with society by using "interpersonal technologies" (Gaggioli et al., 2016). In this regard,

109 Riva and colleagues (Riva et al., 2012) introduced the concept of 'Positive Technologies' directed to 
110 the scientific use of technology to improve users' personal experience, based on positive psychology.

111 More specifically, the authors described three different ways to positively manipulate the users'

112 experiences using technology: i. by structuring personal experience through rules, specific goal 113 interventions, and giving a feedback; ii. by augmenting personal experience through the use of 114 multisensory and multimodal systems; and iii. by replacing personal experience with a fake one (e.g a

115 virtual body). Moreover, the use of such technology for positive psychological interventions must 116 accomplish the following: emotional quality (affect/emotional regulation), engagement and 117 actualization (presence and flow), and connectedness (socialization and networking flow) (Triberti et 118 al., 2016). Thus, rather than positive technologies we should call them transformative technologies for 119 supporting clinical and personal change (Riva et al., 2016).

120 Transformative technologies have been used for affective/emotional regulation (Roth et al., 2019), flow 121 and engagement (Gaggioli et al., 2016; Matamala-Gomez et al., 2020a) psycho-social well-being 122 (Botella et al., 2012), brain training and empowerment (Cipresso et al., 2014), stress and anxiety 123 management (Grassi et al., 2011), and for becoming another through body swapping (Serino et al., $1242016,2019)$. One of the main human-computer interaction systems for transformative experiences to 125 enhance psychological well-being is virtual reality (Riva et al., 2016). VR is an advanced form of 126 human-computer interface that enables participants to interact with an immersive virtual environment 127 which has naturalistic characteristics that the experimenter may manipulate for neuroscientific research 128 or clinical applications (Riva et al., 2016; Spanlang et al., 2014). Through VR systems we can induce 129 the feeling of being embodied in another body (Kilteni et al., 2012a), and modify the morphological 130 characteristic of a virtual body (Kilteni et al., 2012b; Matamala-Gomez et al., 2020b). Following this 131 line, full virtual body illusions through VR has been used to improve body representation in clinical 132 populations with eating disorder patients, specifically in patients with AN (Corno et al., 2018; Keizer 133 et al., 2016; Mölbert et al., 2018; Serino et al., 2019).

\section{$134 \quad 2.2 \quad$ The problem of anorexia}

135 Diagnostic and Statistical Manual of Mental disorders-5 (DSM-5) describes Anorexia Nervosa (AN) 136 as a psychiatric disorder characterized by significant low body weight and the consequent fear of 137 gaining weight (American Psychiatric Association, 2013). A diagnostic criterion for AN is a distortion 138 in perceived body shape and weight, that is a distortion in the patients' body image (American 139 Psychiatric Association, 2013). In this regard, in the last 20 years several studies investigated this 140 disturbance in body perception in patients with AN. One example is the study conducted by Smeets 
141 (1999) in which the author observed that patients with AN considered a thin, normal, overweight or an

142 obese body thinner than what healthy control group considered (Smeets, 1999). However, Tovee and

143 colleagues observed that besides to the reported overestimation body size of the body, AN patients had

144 the tendency to consider lower body mass more attractive than a normal one (Tovee et al., 2000).

145 Further, in this study they also observed that the less the body mass index, the more the overestimation

146 of the body size. Moreover, healthy controls and patients with AN differ for the amount of attention

147 directed to specific parts of the body. One example is the study conducted by Freeman and co-authors

148 (1991) in which healthy controls focused their attention on four areas of the body (face, chest, abdomen,

149 and legs), while patients with AN spent more time observing their legs and abdomen than observing

150 their face (Freeman et al., 1991).

151 From a neurobiological point of view, alterations in the parietal cortices have been linked with spatial 152 and body representations and body ownership (Serino et al., 2015), and with the processing of 153 multisensory integration related to body perception (Riva et al., 2015). Specifically, alterations in the 154 posterior parietal cortex are related to visuospatial processing (Serino et al., 2015). Altogether, these 155 neurological alterations block the online (how or body is right now)-offline (how our body is normally 156 like) interactions to create a coherent representation of our body by the integration of the different 157 sensory signals arriving to our body (Riva, 2014). Hence, according to the latest studies, the integration 158 of new technological systems providing multisensory information such as virtual reality systems can 159 provide new treatment strategies to deal with body image distortions (BID) in patients with AN.

\section{$160 \quad 2.3 \quad$ Virtual reality and anorexia}

161 VR is a useful technology to deal with BID (Döllinger et al., 2019; Riva et al., 2014). In this regard, 162 several studies investigated how to apply VR for both assessment and treatment of the BID in patients 163 with AN (Dakanalis et al., 2017). One of the first studies aimed to investigate the effects of VR in the 164 treatment of eating disorders was conducted by Riva and colleagues (2000), who described the first 165 results of an experimental cognitive therapy in a virtual reality generated environment in two subjects 166 suffering from eating disorders, showing an improved body awareness after the VR exposure. Further, 167 other studies have shown that the combination of a cognitive behavioral therapy with a VR training 168 improves not only the BID, but the eating psychopathology (Perpiñá et al., 2003). The continual 169 improvement of virtual reality systems has led to more sophisticated interventions to treat BID in eating 170 disorders. In addition to the use of VR for the modulation of BID, it has been show that it is possible 171 to change the altered experience of body size in patients with $\mathrm{AN}$ can be changed by inducing a full 
172 body ownership illusion of a virtual body, even for highly emotional body parts (e.g. hips, abdomen)

173 (Keizer et al., 2016). Moreover, VR can be also used to assess self-construction processes in patients

174 with AN. In relation to this, a well-validated VR intervention was used to study the presence of deficits

175 in the egocentric, and allocentric referring frames processing in 12 patients with AN compared with 12

176 healthy controls (Cipolletta et al., 2017). Moreover, this study reported significant impairments in

177 spatial abilities, unidimensional constructing, and an exaggerated construction of the self as perceived

178 by their selves and others while assessing AN in a VR environment [38]. Virtual reality has been also

179 used to generate realistic virtual bodies, through a three-dimensional body scan, to assess BID in AN

180 (Mölbert et al., 2018). Interestingly, Mölbert and colleagues observed that BID in AN may be driven

181 by a mental distorted representation toward the desired body, instead of an overestimation of their

182 actual body weight. Finally, Serino and co-authors used FBI in an immersive VR environment within

183 a three-session VR body-swapping training protocol in one patient with AN to effectively monitor

184 changes of multisensory information perception in body integration, and to test the use of such VR

185 protocol as a driver to promote body representation changes in patients with AN (Serino et al., 2019).

186 In this last study, a reduction in BID and an improvement in the estimation of the patient body size

187 were observed after three session of a body-swapping VR protocol (Serino et al., 2019).

\subsection{Virtual reality, usability and user experience}

189 Traditionally, studies on VR environments have focused on the ability that this kind of environments

190 have to influence peoples' perception (Riva et al., 2019), emotions (Botella et al., 2007), motivations

191 and actions (Slater and Sanchez-Vives, 2016): elements like these make up the criteria for effectiveness

192 of the system, that is if the users can reach their aim with an adequate level of accuracy and

193 completeness within the system ${ }^{1}$. More recently, another criterion of appropriateness emerged: User

194 Experience (UX). UX is considered to be a multi-dimensional construct (Hinderks et al., 2019) that

195 includes “a person's perceptions and responses that result from the use or anticipated use of a product,

196 system or service". Perceptions and responses are a general term indicating that perceptions, emotions,

197 cognitions, motivations, expectations, and actions, all play a role in determining the persons'

198 experience of a system (Winograd and Flores, 1987) before, during, and after the interaction, as

199 indicated by the International Standard Organisation ${ }^{1}$. ${ }^{1}$ https://www.iso.org/obp/ui/\#iso:std:iso:9241:-210:ed-1:v1:en

200 Usability is one of the cornerstones of UX and includes the optimization of the user interface and the 201 perception of ease-of-use and learnability (Nielsen, 1993). In this perspective, elements such as 202 breakdowns and focus shifts (Steed et al., 2003) are considered usability criteria: they indicate a 
misalignment between the users' actions and the system, which causes the technology to become nontransparent and the user to engage in higher cognitive processes to identify the error (Steed et al., 2003). While usability aspects are important, and are necessary for designing an optimal user experience, they are not sufficient to guarantee a good user experience: if $\mathrm{UX}$ is to be considered in a holistic perspective (Pallot and Pawar, 2012), it has to take into consideration the previously mentioned psychological facets of the users, usability and effectiveness of the system.

209 The present pilot study aims to test the effectiveness of a body-swapping VR-based system in terms of

210 affective responses and embodiment scores, and to evaluate its usability and user experience using a 211 quality-quantitative holistic UX perspective.

\section{$2123 \quad$ Methods}

\section{$213 \quad 3.1 \quad$ Participants}

214 Five Italian young women diagnosed of AN following the criteria in the DSM-V participated in this 215 study (age $=17 \pm 1,87)$. All five patients with AN presented a BMI $<16(\mathrm{BMI}=15,95 \pm 0,61)$. Presence 216 of neurological pathologies, history of brain or traumatic brain injury, cognitive deficits and presence 217 of other psychiatric disorders as a comorbidity of the AN were considered as exclusion criteria. The 218 parents of the all five participants signed a consent form to allow the patients to participate in this 219 study. The study was approved by the local ethical committee of the Istituto Auxologico Italiano.

\section{$220 \quad 3.2 \quad$ Study design}

221 The VR-based protocol consisted of four VR experimental sessions of one hour each, taking place 222 during two weeks (two VR sessions per week). Each VR experimental session consisted of two parts: 223 in the first part the patients observed the virtual body from an egocentric (first person perspective) 224 view, in the second part from an allocentric (third person perspective) one. This process, generated the body-swapping illusion induced through synchronous visuo-motor correlations between the real and the virtual body. The presentation of the allocentric or the egocentric perspective of the virtual body was counterbalanced among the patients and sessions. Furthermore, the body mass index (BMI) of the virtual body was changed throughout the VR sessions. In the first VR session, the BMI was the same as the real BMI of the patients with AN $(<16)$, and then this was increased throughout the experimental session until reaching the considered normal BMI (18.5). Further, the autobiographical recall emotion- 
231 induction technique (Chirico et al., 2018) was used: patients were asked to recall negative emotional 232 episode of their life related to the low BMI, and a positive emotional recall related to the normal BMI.

233 Before and after the experimental sessions, patients underwent a baseline assessment in which attitude, 234 cognitive, affective, and perceptive variables related with their clinical condition were tested. In order 235 to evaluate the usability and user experience of this VR-based system, during the VR experimental 236 session the following techniques and tests were used: The think-aloud protocol (Boren and Ramey, 2000), which consist in semi-directed and undisturbed users' verbalization while using and interacting with the VR set-up, providing useful information for interpreting and determining verbal data to identify system deficiencies; Embodiment assessment (Longo et al., 2008), during the experimental session after the exposure of each virtual body condition the level of embodiment, (the sense of body ownership, self-location, and agency toward the virtual body) was assessed by using three different questions in which patients had to indicate how much they agreed or disagreed with each statement in a 7-point Likert scale, from $-3=$ totally disagree to $+3=$ totally agree. The embodiment questions were 244 the following: i) ownership: "I feel that the virtual body is my body"; ii) location: "I feel that my real body is at the same location of the virtual body"; iii) agency: "I feel that I can move freely if I want". Then, after the VR experience, we assessed the overall VR-Experience in terms of ownership, agency, and self-location, with a full 16-items VR-Experience Questionnaire seven-point Likert type from 1: 'totally disagree' to 7: 'totally agree', adapted from (Longo et al., 2008), and the user experience with the User Experience Questionnaire (UEQ-S) seven-point Liker type from -3 (horribly bad) to +3 (extremely good) for each of the 26 items of the questionnaire (Schrepp et al., 2017). User experience after the VR exposure was assessed in session 1 and session 4. In order to simplify the results obtained in the 26-item UEQ, the scales of the UEQ can be grouped into pragmatic quality (Perspicuity,

253 Efficiency, Dependability) and hedonic quality (Stimulation, Novelty). Pragmatic quality describes task related quality aspects, hedonic quality the non-task related quality aspects. Finally, in order to assess the affective state of the patients before and after the VR-protocol we used the short-form of Postive and Negative Affect Schedule (PANAS) (Thompson, 2007). The short-form of PANAS is a 10 item scale (five positive affective items and 5 negative affective items), in which patients have to indicate on a five-point Likert scale how much they agree (5) or disagree (1) are with each statement of the test, in which the maximum score for either the positive or negative items is 25 points (the higher the score the higher the positive or negative affective state). A 4-week follow-up qualitative interview was conducted to verify the final user experience evaluation and the overall experience of the proposed protocol. 
264 A head-mounted display (HDM, Oculus Rift) with a resolution of 2160x1200 pixels per eye in a 265 PenTile OLED panel and with a refresh rate displayed at $90 \mathrm{~Hz}$ to show the virtual environment, which was programmed with Unity version 3.1 (Unity Technologies, San Francisco) was used. The HMD was attached to a laptop computer with an HDMI. The virtual body was taken from the (Unity Library) and configured to match the gender (female) of the participants. In order to induce FBI during the experimental session, synchronous visuomotor stimulations were used, with the virtual body reproducing the real movements of the participants simultaneously. To this aim a motion tracker device connected to the computer a Microsoft Kinect sensor xbox one (www.xbox.com/en-US/kinect) was used. Figure1 A, B.

\subsection{VR session procedure}

275 Patients with AN were asked to stand in front of the computer. During the experiment, patients wore the HMD and receive verbal instructions from the experimenter to follow the experimental session, and to generate the think-aloud and sense of embodiment toward the virtual body. Once the patients put on the HMD, the experimenter asked the patients to look down to their feet, where instead of their real body, they would observe a virtual body co-located with their real body from a first-person perspective (egocentric view) (see figure 1 C, D). Once the patients observed the virtual body and the virtual environment, the experimenter asked them to start the think-aloud while slowly moving their arms upwards, in order to experiment with their virtual body, and to visually explore the virtual environment. Finally, patients were asked to co-locate their real body aligned with the virtual one in order to encourage the embodiment. At this point, patients were asked to verbally express what they felt and their sensations. All patients' responses were audio recorded by the experimenters. Once the think-aloud was concluded VR experimental session started. Each VR experimental session was composed of four different parts: 1) Embodiment phase, 2) Virtual body perspective, 3) Modification of the BMI, and 4) Autobiographical recall. During the embodiment phase (1), embodiment toward the virtual body using synchronous visuo-motor correlations was induced (Kokkinara and Slater, 2014). The experimenters asked the patients to co-locate their real body with the virtual one and not move from that position until the end of the VR session. Then, the experimenter asked the patients to

292 slowly move their real arms from the floor to the ceiling, and slowly repeat this task for three-four 
293 times. Then the patients observed how their virtual arms moved synchronously at the same time and in 294 the same direction, inducing a sense of agency toward the virtual body movements. During the

A)

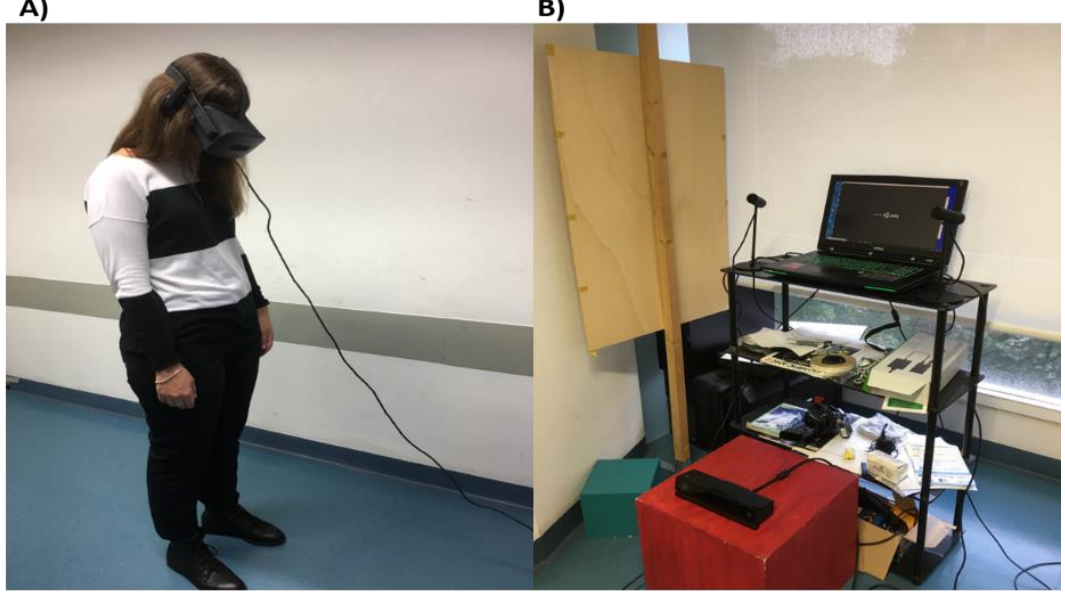

c)

D)

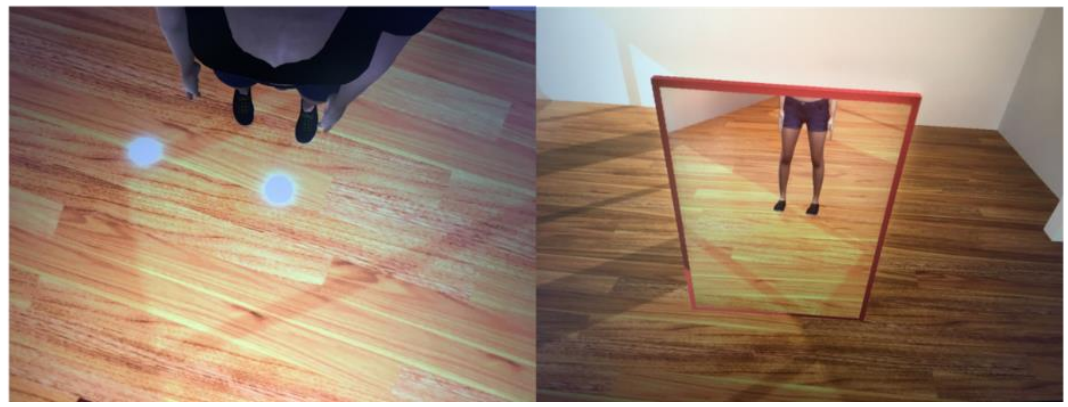

295 embodiment phase, the BMI of the virtual body was different for each experimental session: in session 296 one and two the BMI of the virtual body was equal to the patients with AN real BMI $(<16)$, in session three the BMI of the virtual body increased to 17.5 (closer the normal BMI), and in the

298 fourth session it increased again to 18.5 (normal and healthy BMI). In the virtual body perspective 299 phase (2), the experimenter changed the perspective view of the virtual body in an allocentric or egocentric perspective depending of the session (see study design methods). After changing the virtual

301 body perspective (3), the experimenters asked the patients to verbally indicate the virtual body 302 dimension until it reached their desired body size, and then to their perceived real body size 303 for both allocentric and egocentric virtual body perspectives, and the experimenter modified the 304 avatars' BMI through the VR computer interface accordingly (visuomotor correlations were re305 induced after each virtual body exposure). After the observation of each virtual body condition 306 embodiment toward the virtual body was assessed. Finally (4), the patients had to associate their virtual 307 body to a negative (session 2 and 3) or positive (session 4) event of their life depending on the session 308 (see study design section). At the end of the experimental session patients had to fill in a full 309 embodiment questionnaire. 
310 Figure 1. Virtual reality set-up and body swapping illusion. A) Participant wearing the HMD. B) Virtual reality set-up

311 composed of a laptop, Microsoft Kinect tracking system, and Oculus sensors to track body position. C) Egocentric view

312 perspective of the virtual body. D) Allocentric view perspective of the virtual body.

\section{Data handling}

314 All statistical tests for the repeated measures of the study (embodiment questions after each condition 315 and data from PANAS test) were performed in Stata 13 (StataCorp LP, College Station, TX, USA).

316 These measures are therefore a mixed-effects design, with virtual body BMI (real/desired) and Body

317 perspective (egocentric/allocentric) as fixed-effects, and ownership, location, and agency scoring as

318 the dependent variables, with random effects over the "individual subjects". Differences in

319 embodiment questions across conditions with multilevel mixed-effect ordered logistic regression test

320 (the 'meologit' function in Stata) were analyzed. Furthermore, a multilevel mixed-effects linear

321 regression test (the 'mixed' function in Stata) was performed to observe the effects of the VR

322 intervention on the positive and negative affect of the patients with fixed-effect on Time (pre-VR/post-

323 VR) and positive or negative PANAS scoring as the dependent variable. Due to the small sample size

324 of the study $(n=5)$, we show a descriptive statistical of data for the UEQ. Results from the UEQ were

325 analyzed by using the UEQ data analysis tool (version 4) (Schrepp, 2015). Qualitative thematic analysis

326 was used to identify recurrent themes in the interviews. The authors used interview notes and

327 recordings to report the main patterns within the data. Recordings of the thinking aloud section of the

328 study were generated and analyzed in a concurrent ethnography perspective, a specific sub-

329 methodology of focused ethnography (Martinez Perez and Camas Baena, 2016) adapted for

330 technological design and to support usability.

\section{Results}

\section{$332 \quad 5.1 \quad$ Embodiment questionnaire scoring}

333 During the VR session, we assessed the level of embodiment after the exposure to each virtual body

334 condition (real vs. desired virtual body), from an egocentric or allocentric perspective. Further, at the

335 end of each VR session we assessed the overall level of embodiment. Patients reported good levels of

336 embodiment when the BMI of the virtual body to their perceived real BMI was changed. Specifically,

337 patients reported significantly higher levels of ownership ( $\mathrm{z}=4.98, \mathrm{p}<0.0001)$, location $(\mathrm{z}=-2.27$,

$338 \mathrm{p}=0.023)$, and agency $(\mathrm{z}=-2.05, \mathrm{p}=0.041)$ toward the virtual body when they were embodied in a virtual

339 body with their perceived real BMI, than when they were embodied in a virtual body with their desired 
340 BMI (Figure 2). However, no differences were found for ownership $(\mathrm{z}=1.41, \mathrm{p}=0.159)$, location $(\mathrm{z}=-$ $3410.56, \mathrm{p}=0.577)$, and agency $(\mathrm{z}=-1.07, \mathrm{p}=0.286)$ while observing the virtual body form an egocentric 342 (1PP) or allocentric point of view (3PP). Figure 2.

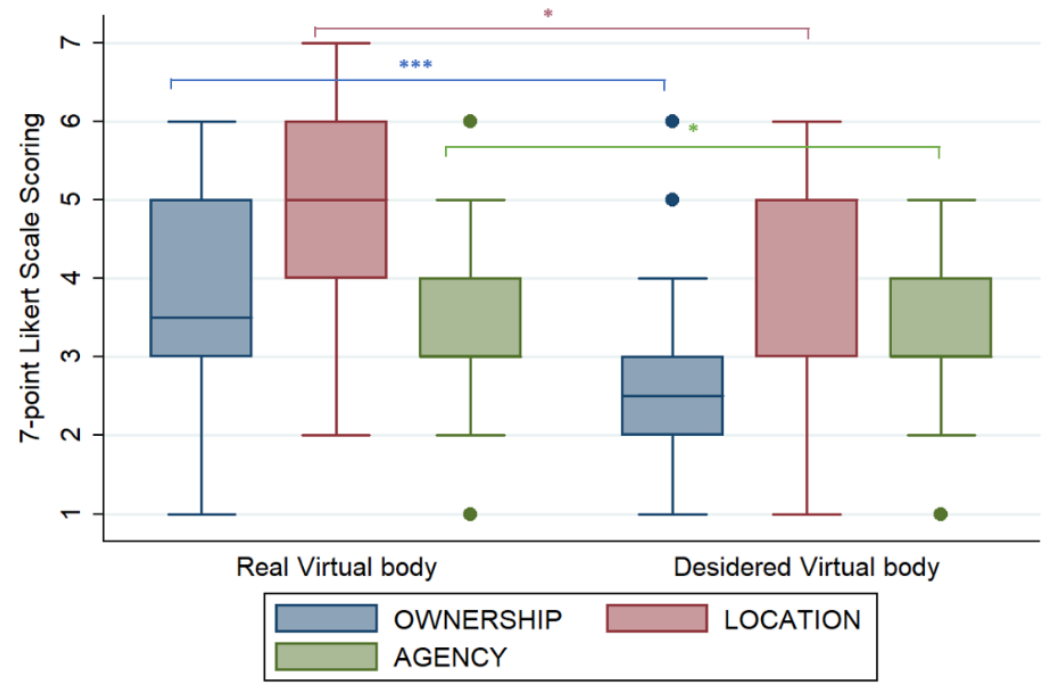

343 Figure 2. Embodiment levels increase with the perceived real BMI of the virtual body. Ownership, location and agency 344 ratings after observing the virtual body with the real perceived BMI show higher scores compared to the observation of the 345 virtual body with the desired BMI. Boxplots show medians (horizontal lines), interquartile ranges (IQR; boxes) data outside 346 1.5xIQR (whiskers) and outliers (o). *p<0.05, **p<0.01, ***p $<0.001$.

347 In order to simplify the results obtained in the 16-item embodiment questionnaire four different 348 variables were created by grouping the items regarding to ownership questions, ownership control 349 questions, agency questions, and location questions of the questionnaire. The outcomes obtained from 350 the embodiment questionnaire show a reported median level of embodiment (median=3) for patients

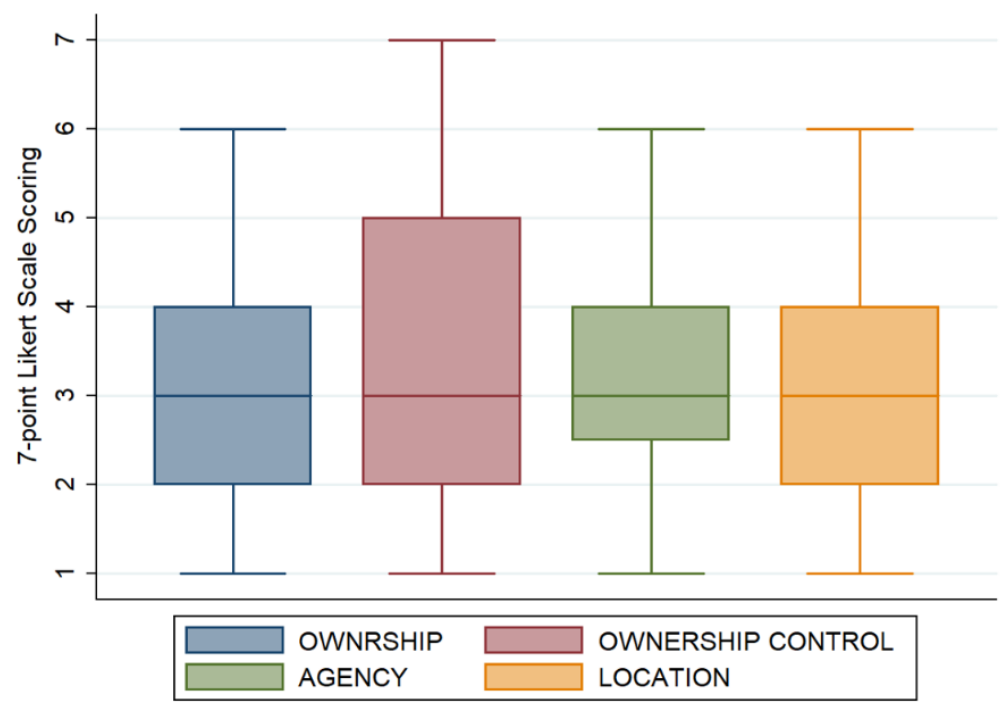


351 after the whole VR experience. No significant differences were found for ownership and ownership 352 control questions; however, ownership control variable show a higher scoring than ownership variable 353 Figure 3.

354 Figure 3. Embodiment ratings after VR exposure. Questionnaire ratings after the virtual reality exposure show that 355 patients reported median levels of ownership, ownership control, agency and location of the virtual body, ownership scores 356 increased in the ownership control questions. Boxplots show medians (horizontal lines), interquartile ranges (IQR; boxes) 357 data outside 1.5xIQR (whiskers) and outliers (o).

\section{$358 \quad 5.2 \quad$ PANAS and user experience questionnaire}

359 The reported levels of the positive and negative affective state of the patients before and after each VR session show a statistically significant difference between pre-VR and post-VR after the second VR session for the positive affective scoring after the VR exposure $(\mathrm{z}=-2.50, \mathrm{p}=0.012)$. Nevertheless, patients reported significant lower negative affective levels after the four VR session compared with levels reported before the VR exposure $(z=2.74, p=0.006)$. See figure 4.

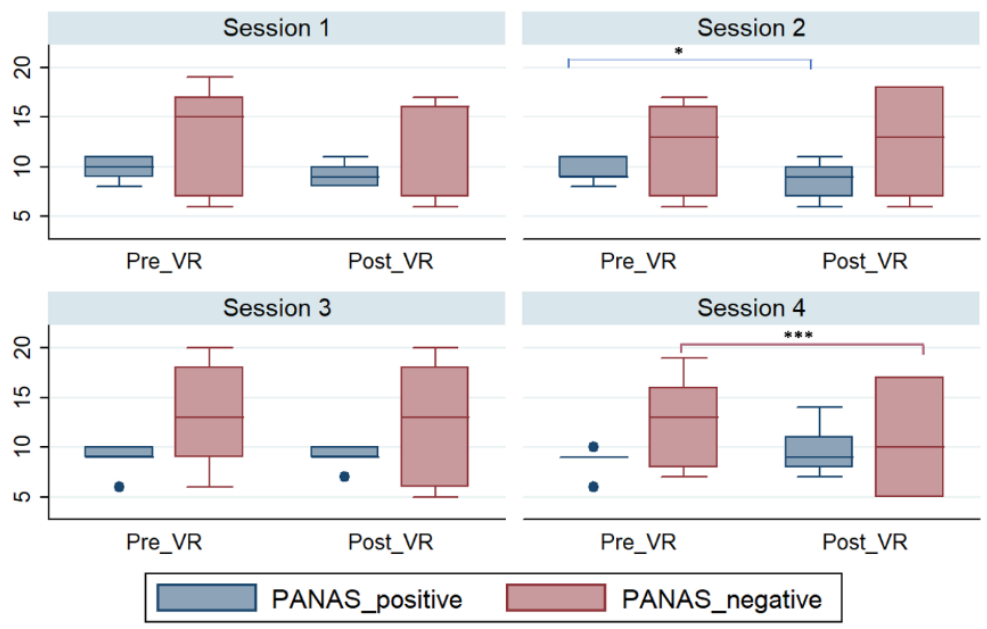

364 Figure 4. Negative affective state increase after session 2 and positive affective state increase after session 4 . The graph shows the positive and negative affective state ratings differences before and after the VR exposure in each session (from session 1 to session 4). Boxplots show medians (horizontal lines), interquartile ranges (IQR; boxes) data outside 1.5xIQR (whiskers) and outliers (o). $* \mathrm{p}<0.05, * * \mathrm{p}<0.01, * * * \mathrm{p}<0.001$.

\subsection{User experience outcomes after VR exposure}

369 The results obtained in the UEQ showed that patients perceived the system as more attractive after the 370 VR exposure in session 1 (mean $\pm \mathrm{SD}=0,533 \pm 0,462)$ than after session 4 (mean $\pm \mathrm{SD}=0,00 \pm 0,593)$. On 371 the other hand, patients perceived the system more familiar and easier to use after session 4 
$372($ mean $\pm \mathrm{SD}=1,125 \pm 0,721)$ than after session 1 (mean $\pm \mathrm{SD}=0,950 \pm 0,596)$. Average scoring for novelty 373 were higher after session $4($ mean $\pm \mathrm{SD}=1,688 \pm 0,943)$ than after session $1($ mean $\pm \mathrm{SD}=1,550 \pm 1,229)$, 374 figure 5.
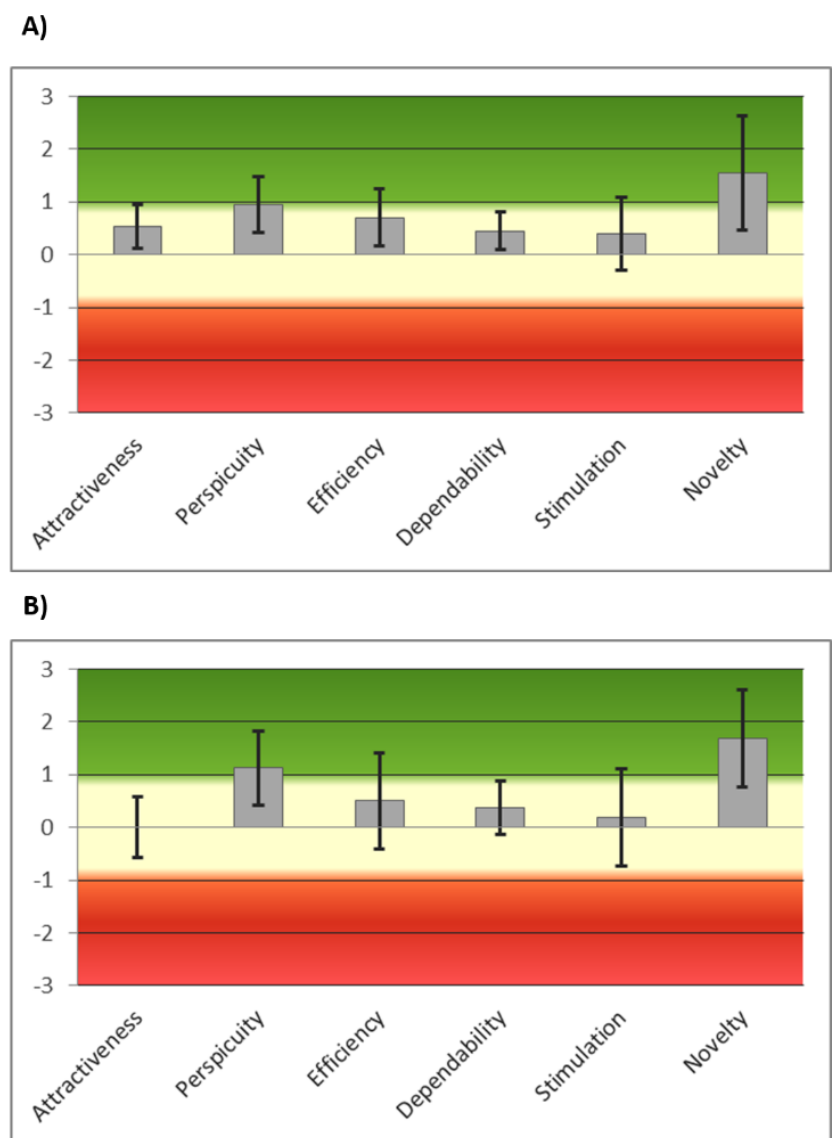

375 Figure 5. User experience after VR exposure in session one and four. (A) UEQ ratings after VR exposure in session 1, were high for attractiveness and novelty. (B) UEQ ratings after VR exposure in session 4, were high for perspicuity and novelty. The bar chart shows the media and standard deviations obtained in the six different qualities of the VR-system.

\subsection{Thinking aloud}

379 The thinking aloud technique revealed that no element in the virtual environment gained more attention

380 than the others: two patients first focused on the virtual body and two on the virtual environment. The 381 virtual environment is described by most patients in details, giving attention especially to the interior 382 of the room. The virtual world outside the window is not given much consideration. The description of 383 the virtual body is more critical. $\mathrm{N}=4$ patients used personal nouns and pronouns when describing the 384 virtual body, which indicates a good level of identification with the virtual body ("I saw myself 385 reflected in a mirror"). When there was a break-down between the participants' movements and the 386 virtual bodies' (uncontrollable movements and/or non-responsive virtual limbs) the embodiment failed, 387 as indicated by the participants' switch to third person noun and pronouns ("The virtual body moves 
as I move but sometimes (it does) not" (patient 1), "The virtual foot is not co-located with my real foot, it seems twisted" (patient 3). Overall, patients experienced an average of two break-downs of the illusion per session. Although for some specific aspects of the virtual experience the opinion of the patients was different, patients' reported speech during the thinking aloud phase was consistent among

392 the patients.

\subsection{Patients interview}

394 The following results are extracted from a follow-up interview of three of the patients with AN of the 395 study, one month after the VR experience. First, all patients had previous knowledge of VR but only one patient actually had previous experience with a VR environment used in a clinical setting. One had previously tried a VR-based entertainment system, but only in one occasion. Secondly, we observed that all three patients appreciated the contextual features of the VR room, which was deemed clear and detailed. However, the virtual body generated contrasting feelings: while the patients could tell that it was meant to be them, the virtual body was usually perceived as unfamiliar and not similar enough to their own body. This feeling may be exacerbated by the several breakdowns they experienced when the virtual body movements would not mirror their own. The body similarity between the virtual body and the real body of the patients emerged as particularly relevant especially in the two youngest interviewees, who expressed frustration in not being able to control the body of the avatar body completely, both in its physical aspects and in its limited motion. This fixation on the body is on par with the AN pathology and with another topic that emerged from the interviews, which is the scarce understanding of the goal of the proposed VR experience and the 4-VR sessions. The system itself does not give any hint of the aim of being in the VR and does not give any kind of feedback to the participants, and relies on the research personnel managing the technological set up to give instruction and sense to the experience. This contradicts Nielsen's visibility of system status heuristic [53], which dictates that a system should always keep the user informed of what is happening through timely

412 feedbacks. Since they do not fully understand the aim of the program, participants are also unable to 413 express an opinion on the usefulness of the system and the entire protocol: they entered the study 414 because they were encouraged by the medical personnel who presented it (not the researchers) to their 415 parents. They reported that it does not seem well connected to their healing process and their activities 416 at the hospital. The immersive VR experience is deemed 'weird' and 'strange' at the beginning of the 417 protocol, but participants said that it is easy to get used to. 
418 The 4-session protocol is 'boring' and 'repetitive': participants did not perceive any change in the

419 avatars and in what they were asked to do in the environment. While this may be positive from the

420 research point of view, it is not optimal for the continuing levels of motivations of the users, who may

421 abandon the program or decide not to repeat it. The interviewees said they would continue/re-attend

422 the program due to the fact that it is very innovative and they like the VR aspect of it, which is also the

423 reason they would suggest others to try it. So, the VR systems' novelty and innovation is the main

424 driver for continued use. Another important emerging topic is the lack of sense making regarding the

425 experience in the participants' life and healing process: while one participant said the experience

426 stimulated reflections and it may be useful talking about it during the healing process, the rest of the

427 participants said that they did not discuss it in depth nor with their parents and friends nor with their

428 health care providers. The emotional response they had to both the VR technology and the program in

429 its entirety was also very limited and weak: other than the initial strangeness of VR and the

430 tiresomeness perceived during the program, two participant out of three did not report any particular

431 negative or positive feelings at all, even regarding the emotional recall part. Then, the experience was

432 categorized as a one-off experience which broke the monotony of their daily life. This makes sense in

433 the organizational context the system where was tested, which is a hospital offering Occupational

434 Therapy-based support to patients with AN. To maximize the UX of the full program it may be useful

435 to really include it in the therapeutic process, as experiencing another body is a transformative 436 experience which may include changes at self-level.

\section{Discussion}

438 Immersive virtual reality can induce sense of owning a virtual body by providing synchronous visuo439 motor correlations between the real and the virtual body to the users (Sanchez-Vives et al., 2010).

440 Further, VR can generate the perceptual illusion of swapping toward another fake body (Petkova and

441 Ehrsson, 2008). Such virtual body illusions can be used for clinical proposes to modulate body 442 perception in patients with a body image distortion due to their clinical conditions (Matamala-Gomez 443 et al., 2019). Although some studies investigated the use of embodiment while using VR systems in 444 clinical applications, few studies reported how the characteristics of the user experience is while using 445 immersive VR systems. The present manuscript shows the results obtained in a pilot study conducted 446 with five patients with AN to explore user experience by analysing individual thinking about the system 447 while using it. In addition, the level of embodiment and the affective modulation after the VR exposure 448 was also assessed. Further, a personal interview with each of the patients participating in the study one 
month after the use of the VR system as a follow-up of the personal experience was conducted. The

450 present pilot study shows that the proposed VR-body swapping protocol can be used as a powerful tool

451 in clinical population suffering from body image distortion. The VR-system was able to induce high

452 levels of embodiment and of subcomponents of embodiment: sense of ownership, agency, and self-

453 location, toward a virtual body while being embodied in a virtual body with a real perceived BMI.

454 However, embodiment levels decreased when the patients were embodied in a virtual body with their

455 desired BMI. This result is in line with the study conducted by Serino and co-authors (2019) in which

456 the authors showed how the VR-based body swapping illusion protocol worked as a helpful and useful

457 tool to assess and to modify body distortions in clinical populations. The patients perceived the BMI

458 of their real body as larger than their real BMI and this can be due to the fact that patients identify more

459 with the virtual body presenting their real perceived BMI. In this regard, the fact that patients identify

460 more with the real perceived BMI virtual body shows the body image distortion of the patients

461 participating in our study, as they perceived real BMI is larger than their actual real BMI. Moreover,

462 as in the study conducted by Serino and co-authors, this study confirms that high embodiment levels

463 indicate that body image in clinical population can be re-shaped, with body image distortion by using

464 the here proposed VR-body swapping illusion protocol. Secondly, the proposed VR protocol together

465 with the autobiographical recall technique seems to modulate the affective state of the patients with

466 AN after the VR exposure. This result is not only due to the VR exposure but also to the positive or

467 negative autobiographical recall associated to the virtual body (see VR procedures section). In fact, the negative affective state increased after the VR exposure in session two, in which patients had to associate their observed virtual body (low BMI) with a negative event of their life, and positive affective state increased after the VR exposure in session four, where patients have to associate their observed virtual body (normal BMI) with a positive event of their life. This result is very relevant from a clinical point of view as even though this affective component had been proposed to be used in patients suffering from obesity (Riva, 2011), this is the first time that an affective component was associated with the virtual body observation.

Regarding the usability and the UX of the system, both the UEQ, break down analysis, and the interviews revealed that the participants' evaluation of the experience was sub-optimal: novelty and innovation of the VR system were the positive aspects of the program; on the other hand, the usability of the system was negatively depicted due to the several break-down of the virtual body illusion because of some problems with the tracking movement system and probably also due to latency issues (Friston and Steed, 2014). The results collected form the interviews also explain the embodiment levels 
obtained in the embodiment questionnaire after the VR exposure, in which we observed median levels 482 of ownership, agency, and self-location toward the virtual body. However, the higher scoring obtained 483 in the ownership control variable may be explained because of the clinical condition of the patients 484 presenting a distorted body image that is also present into the virtual environment. This result reflects 485 a limitation in defining the limits of their body, as expected and in keeping with other studies that used 486 virtual embodiment in clinical populations presenting body image distortions (Matamala-Gomez et al., 2018; Mölbert et al., 2018; Serino et al., 2019).

\section{$7 \quad$ Limitations}

A limitation of the present study is the small sample size $(n=5)$ which reduces the generalizability of the results obtained with the proposed VR-body swapping illusion protocol to other clinical populations. However, the obtained data in the study is homogeneous enough to hypothesize that other critical factors will not appear when using this VR system with a larger sample size. Another limitation of this study is that cyber sickness and other psychological constructs such as sense of presence after the use of the VR system were not specifically assessed. However, none of the 5 patients reported signs of cybersickness (Rebenitsch and Owen, 2016) neither in the thinking aloud phase or in the follow-up interviews about the system. Further trials should also consider introducing measures to control for latency aspects (Friston and Steed, 2014).

\section{Conclusion}

The present study demonstrated that the proposed VR-body swapping illusion protocol can be used for both assessment and clinical proposes in clinical populations suffering from AN. Further, the study shows the feasibility to use a low-cost head-mounted display connected to a laptop, through which patients can be immersed in a virtual environment and embodied into a virtual body. The VR system offers the possibility of changing the morphological characteristics of the virtual body (e.g. changing the BMI of the virtual body) and observe it from different perspectives (third- vs. first-person), targeting body image distortion in patients with AN through a powerful and effective tool. Further, embodiment scores show that the system works for its intended purpose of experiencing another body. While we can consider the system effectiveness in inducing embodiment, further iteration of the system and the full program should aim to introduce goal transparency both in and out of the VR system, shorter latency timing and more accurate tracking system, and avatar personalization to improve usability and user experience. 
5119 Conflict of Interest

512 The authors declare that the research was conducted in the absence of any commercial or financial

513 relationships that could be construed as a potential conflict of interest.

\section{$51410 \quad$ Author Contributions}

515 MM-G contributed to the data collection and statistical analyses, bibliographic review, 516 conceptualization, and writing of the manuscript. EB contributed to the data collection, writing and 517 conceptualization of the manuscript. $\mathrm{CM}$ and $\mathrm{AC}$ contributed to the data collection, and 518 conceptualization of the study. OR contributed to the bibliographic review and writing of the 519 manuscript. SS and GC contributed to the conceptualization of the study and design of the virtual 520 reality scenario. NP, AD, CC contributed to the patient collection and in the conceptualization of the 521 clinical aspects of the study. GR, and FM contributed to the supervision of the manuscript. All the 522 authors approved the final version of the manuscript for submission.

\section{$523 \quad 11 \quad$ Funding}

524 This paper was supported by the Italian MIUR (Ministerio dell'Università e della Ricerca Italiana). 525 Research project "Unlocking the memory of the body: Virtual Reality in anorexia nervosa (Grant $\left.526 \quad \mathrm{~N}^{\circ} .201597 \mathrm{WTTM}\right)$.

12 Reference

528 American Psychiatric Association (2013). "DSM-5 Diagnostic Classification," in Diagnostic and $529 \quad$ Statistical Manual of Mental Disorders doi:10.1176/appi.books.9780890425596.x00diagnosticclassification.

531 Boren, M. T., and Ramey, J. (2000). Thinking aloud: Reconciling theory and practice. IEEE Trans. Prof. Commun. doi:10.1109/47.867942.

533 Botella, C., García-Palacios, A., Villa, H., Baños, R. M., Quero, S., Alcañiz, M., et al. (2007). Virtual reality exposure in the treatment of panic disorder and agoraphobia: A controlled study. Clin. Psychol. Psychother. 14, 164-175. doi:http://dx.doi.org/10.1002/cpp.524. 
Botvinick, M., and Cohen, J. (1998). Rubber hands "feel" touch that eyes see [8]. Nature 391, 756. doi:10.1038/35784.

Burgess, N. (2019). Positive psychology: the scientific and practical explorations of human strengths. Fourth edition. Educ. Psychol. Pract. 35, 354-355. doi:10.1080/02667363.2019.1602302.

Chirico, A., Cipresso, P., and Gaggioli, A. (2018). Psychophysiological Specificity of Four Basic Emotions Through Autobiographical Recall and Videos. in Lecture Notes of the Institute for

Cipolletta, S., Malighetti, C., Serino, S., Riva, G., and Winter, D. (2017). Intrapersonal, interpersonal, and physical space in anorexia nervosa: a virtual reality and repertory grid investigation.

Cipresso, P., Albani, G., Serino, S., Pedroli, E., Pallavicini, F., Mauro, A., et al. (2014). Virtual multiple errands test (VMET): A virtual reality-based tool to detect early executive functions

Corno, G., Serino, S., Cipresso, P., Baños, R. M., and Riva, G. (2018). Assessing the Relationship Between Attitudinal and Perceptual Component of Body Image Disturbance Using Virtual Reality. Cyberpsychology, Behav. Soc. Netw. 21, 679-686. doi:10.1089/cyber.2018.0340.

Dakanalis, A., Manzoni, G. M., Castelnuovo, G., Riva, G., and Clerici, M. (2017). Towards novel paradigms for treating dysfunctional bodily experience in eating disorders. Eat. Weight Disord. 22, 373-375. doi:10.1007/s40519-017-0361-5.

Döllinger, N., Wienrich, C., Wolf, E., and Latoschik, M. E. (2019). ViTraS-Virtual Reality Therapy beholder: Processing body shape information in anorexic and bulimic patients. Int. J. Eat. Disord. 10, 709-714. doi:10.1002/1098-108X(199111)10:6<709::AID- 
566

568

569

570

571

572

573

574

575

576

577

578

579

580

581

582

Friston, S., and Steed, A. (2014). Measuring latency in virtual environments. IEEE Trans. Vis. Comput. Graph. 20, 616-625. doi:10.1109/TVCG.2014.30.

Gaggioli, A., Chirico, A., Triberti, S., and Riva, G. (2016). Transformative interactions: Designing positive technologies to foster self-transcendence and meaning. Annu. Rev. CyberTherapy Telemed. 14, 169-176.

Grassi, A., Gaggioli, A., and Riva, G. (2011). New technologies to manage exam anxiety. Annu. Rev. CyberTherapy Telemed. 9, 45-49.

Hinderks, A., Schrepp, M., Domínguez Mayo, F. J., Escalona, M. J., and Thomaschewski, J. (2019). Developing a UX KPI based on the user experience questionnaire. Comput. Stand. Interfaces. doi:10.1016/j.csi.2019.01.007.

Keizer, A., Van Elburg, A., Helms, R., and Dijkerman, H. C. (2016). A virtual reality full body illusion improves body image disturbance in anorexia nervosa. PLoS One 11, e0163921. doi:10.1371/journal.pone.0163921.

Kilteni, K., Groten, R., and Slater, M. (2012a). The Sense of Embodiment in Virtual Reality. Presence Teleoperators Virtual Environ. 21, 373-387. doi:10.1162/PRES_a_00124.

Kilteni, K., Normand, J. M., Sanchez-Vives, M. V., and Slater, M. (2012b). Extending body space in immersive virtual reality: A very long arm illusion. PLoS One 7, e40867. doi:10.1371/journal.pone.0040867.

Kokkinara, E., and Slater, M. (2014). Measuring the effects through time of the influence of visuomotor and visuotactile synchronous stimulation on a virtual body ownership illusion. Perception 43, 43-58. doi:10.1068/p7545.

Longo, M. R., Schüür, F., Kammers, M. P. M., Tsakiris, M., and Haggard, P. (2008). What is embodiment? A psychometric approach. Cognition 107, 978-998. doi:10.1016/j.cognition.2007.12.004.

Martinez Perez, A., and Camas Baena, V. (2016). Etnography of empowerment in Europe and America: designing the future with communities. EMPIRIA, 47-70. 
593

595

596

597

598

Maselli, A., and Slater, M. (2013). The building blocks of the full body ownership illusion. Front. Hum. Neurosci. 7. doi:10.3389/fnhum.2013.00083.

Matamala-Gomez, M., Donegan, T., Bottiroli, S., Sandrini, G., Sanchez-Vives, M. V., and Tassorelli, C. (2019). Immersive Virtual Reality and Virtual Embodiment for Pain Relief. Front. Hum. Neurosci. 13, 279. doi:10.3389/fnhum.2019.00279.

Matamala-Gomez, M., Gonzalez, A. M. D., Slater, M., and Sanchez-Vives, M. V. (2018). Decreasing pain ratings in chronic arm pain through changing a virtual body: different strategies for different pain types. J. Pain. doi:10.1016/J.JPAIN.2018.12.001.

Matamala-Gomez, M., Maisto, M., Montana, J. I., Mavrodiev, P. A., Baglio, F., Rossetto, F., et al. (2020a). The role of engagement in teleneurorehabilitation: A systematic review. Front. Neurol. 11. doi:10.3389/fneur.2020.00354.

Matamala-Gomez, M., Nierula, B., Donegan, T., Slater, M., and Sanchez-Vives, M. V. (2020b). Manipulating the Perceived Shape and Color of a Virtual Limb Can Modulate Pain Responses. J. Clin. Med. 9, 291. doi:10.3390/jcm9020291.

Mölbert, S. C., Thaler, A., Mohler, B. J., Streuber, S., Romero, J., Black, M. J., et al. (2018). Assessing body image in anorexia nervosa using biometric self-avatars in virtual reality: Attitudinal components rather than visual body size estimation are distorted. Psychol. Med. 48, 642-653. doi:10.1017/S0033291717002008.

Montana, J. I., Gomez, M. M., Maisto, M., Mavrodiev, P. A., Cavalera, C. M., Diana, B., et al. (2020). The Benefits of emotion Regulation Interventions in Virtual Reality for the Improvement of Wellbeing in Adults and Older Adults : A Systematic Review. J. Clin. Med. 9, 1-22. doi:10.3390/jcm9020500.

Neyret, S., Bellido Rivas, A. I., Navarro, X., and Slater, M. (2020). Which Body Would You Like to Have? The Impact of Embodied Perspective on Body Perception and Body Evaluation in Immersive Virtual Reality. Front. Robot. AI 7, 31. doi:10.3389/frobt.2020.00031.

Nielsen, J. (1993). Usability Engineering. Morgan Kaufmann Publ. Inc. 
620

621

622

623

624

625

626

627

628

629

630

631

632

634

635

636

637

638

639

640

641

642

643

644

Pallot, M., and Pawar, K. (2012). A holistic model of user experience for living lab experiential design. in 2012 18th International Conference on Engineering, Technology and Innovation, ICE 2012 - Conference Proceedings doi:10.1109/ICE.2012.6297648.

Parsons, T. D. (2015). Virtual reality for enhanced ecological validity and experimental control in the clinical, affective and social neurosciences. Front. Hum. Neurosci. 9. doi:10.3389/fnhum.2015.00660.

Perpiñá, C., Marco, J. H., Botella, C., and Baños, R. (2003). Treatment of body image disturbances in eating disorders by means of virtual reality: A case report. Psicol. Conductual 11.

Petkova, V. I., and Ehrsson, H. H. (2008). If I were you: Perceptual illusion of body swapping. PLoS One 3. doi:10.1371/journal.pone.0003832.

Rebenitsch, L., and Owen, C. (2016). Review on cybersickness in applications and visual displays. Virtual Real. doi:10.1007/s10055-016-0285-9.

Riva, G. (2011). The key to unlocking the virtual body: Virtual reality in the treatment of obesity and eating disorders. J. Diabetes Sci. Technol. 5, 283-292. doi:10.1177/193229681100500213.

Riva, G. (2014). Out of my real body: Cognitive neuroscience meets eating disorders. Front. Hum. Neurosci. 8. doi:10.3389/fnhum.2014.00236.

Riva, G., Baños, R. M., Botella, C., Mantovani, F., and Gaggioli, A. (2016). Transforming experience: The potential of augmented reality and virtual reality for enhancing personal and clinical change. Front. Psychiatry 7. doi:10.3389/fpsyt.2016.00164.

Riva, G., Baños, R. M., Botella, C., Wiederhold, B. K., and Gaggioli, A. (2012). Positive technology: Using interactive technologies to promote positive functioning. Cyberpsychology, Behav. Soc. Netw. 15, 69-77. doi:10.1089/cyber.2011.0139.

Riva, G., Gaudio, S., and Dakanalis, A. (2014). I'm in a virtual body: A locked allocentric memory may impair the experience of the body in both obesity and anorexia nervosa. Eat. Weight Disord. 19, 133-134. doi:10.1007/s40519-013-0066-3. 
645

646

647

648

649

650

651

652

653

654

655

656

657

658

659

660

661

662

663

664

665

666

667

668

669

670

671

Riva, G., Gaudio, S., and Dakanalis, A. (2015). The neuropsychology of self-objectification. Eur. Psychol. 20, 34-43. doi:10.1027/1016-9040/a000190.

Riva, G., Mantovani, F., Capideville, C. S., Preziosa, A., Morganti, F., Villani, D., et al. (2007). Affective Interactions Using Virtual Reality: The Link between Presence and Emotions. CyberPsychology Behav. doi:10.1089/cpb.2006.9993.

Riva, G., Wiederhold, B. K., and Mantovani, F. (2019). Neuroscience of Virtual Reality: From Virtual Exposure to Embodied Medicine. Cyberpsychology, Behav. Soc. Netw. 22, 82-96. doi:10.1089/cyber.2017.29099.gri.

Roth, D., Stauffert, J.-P., and Latoschik, M. E. (2019). “Avatar Embodiment, Behavior Replication, and Kinematics in Virtual Reality," in VR Developer Gems, 321-346. doi:10.1201/b21598-17.

Sanchez-Vives, M. V, Spanlang, B., Frisoli, A., Bergamasco, M., and Slater, M. (2010). Virtual hand illusion induced by visuomotor correlations. PLoS One 5, e10381. doi:10.1371/journal.pone.0010381.

Schrepp, M. (2015). User Experience Questionnaire Handbook. Available at: https://www.researchgate.net/profile/Martin_Schrepp/publication/281973617_User_Experience _Questionnaire_Handbook/links/56004c7008aeafc8ac8c64bb.pdf [Accessed October 14, 2019].

Schrepp, M., Hinderks, A., and Thomaschewski, J. (2017). Design and Evaluation of a Short Version of the User Experience Questionnaire (UEQ-S). Int. J. Interact. Multimed. Artif. Intell. doi:10.9781/ijimai.2017.09.001.

Seligman, M. E., and Csikszentmihalyi, M. (2000). Positive Psychology: An Introduction. American Psychologist. Am. Psychol.

Serino, S., Dakanalis, A., Gaudio, S., Carrà, G., Cipresso, P., Clerici, M., et al. (2015). Out of body, out of space: Impaired reference frame processing in eating disorders. Psychiatry Res. 230, $732-$ 734. doi:10.1016/j.psychres.2015.10.025.

Serino, S., Pedroli, E., Keizer, A., Triberti, S., Dakanalis, A., Pallavicini, F., et al. (2016). Virtual reality body swapping: A tool for modifying the allocentric memory of the body. Cyberpsychology, Behav. Soc. Netw. 19, 127-133. doi:10.1089/cyber.2015.0229. 
672 Serino, S., Polli, N., and Riva, G. (2019). From avatars to body swapping: The use of virtual reality 673 for assessing and treating body-size distortion in individuals with anorexia. J. Clin. Psychol. 75, 674 313-322. doi:10.1002/jclp.22724.

675

676

Slater, M. (2009). Place illusion and plausibility can lead to realistic behaviour in immersive virtual environments. Philos. Trans. R. Soc. B Biol. Sci. 364, 3549-3557. doi:10.1098/rstb.2009.0138.

Slater, M., and Sanchez-Vives, M. V. (2016). Enhancing our lives with immersive virtual reality. Front. Robot. AI 3, 74. doi:10.3389/frobt.2016.00074.

Smeets, M. A. M. (1999). Body size categorization in anorexia nervosa using a morphing instrument. Int. J. Eat. Disord. 25, 451-455. doi:10.1002/(SICI)1098-108X(199905)25:4<451::AIDEAT10>3.0.CO;2-0.

Spanlang, B., Normand, J.-M., Borland, D., Kilteni, K., Giannopoulos, E., PomÃ@s, A. s, et al. (2014). How to Build an Embodiment Lab: Achieving Body Representation Illusions in Virtual Reality. Front. Robot. AI 1. doi:10.3389/frobt.2014.00009.

Steed, A., Brogni, A., and Vinayagamoorthy, V. (2003). Breaks in Presence as Usability Criteria. Computer (Long. Beach. Calif).

Thompson, E. R. (2007). Development and validation of an internationally reliable short-form of the Positive and Negative Affect Schedule (PANAS). J. Cross. Cult. Psychol. doi:10.1177/0022022106297301.

Tovee, M. J., Emery, J. L., and Cohen-Tovee, E. M. (2000). The estimation of body mass index and physical attractiveness is dependent on the observer's own body mass index. Proc. R. Soc. B Biol. Sci. 267, 1987-1997. doi:10.1098/rspb.2000.1240.

Triberti, S., Chirico, A., and Riva, G. (2016). "New technologies as opportunities for flow experience: A framework for the analysis," in Flow Experience: Empirical Research and Applications, 249-263. doi:10.1007/978-3-319-28634-1_16.

Winograd, T., and Flores, F. (1987). On understanding computers and cognition: A new foundation for design. A response to the reviews. Artif. Intell. doi:10.1016/0004-3702(87)90026-9. 
699 